\title{
Fungi isolated in blood cultures in Ege University Medical Faculty, Childrens Hospital between 2008-2017
}

Zümrüt Şahbudak Bal ${ }^{1}$, Gizem Güner ${ }^{1}$, Dilek Yeşim Metin², Ferda Özkınay ${ }^{1}$, Zafer Kurugöl ${ }^{1}$, Süleyha Hilmioğlu Polat ${ }^{2}$

${ }^{1}$ Department of Pediatrics, ${ }^{2}$ Department of Medical Microbiology, Faculty of Medicine, Ege University, Izmir, Turkey.

Although the spectrum of fungi causing bloodstream fungal infections continues to expand, Candida spp. remains responsible for the majority of these cases. The distribution of Candida species varies according to countries, regions and also institutions.

In this study, we evaluated retrospectively the distribution of Candida species yielded from blood cultures of hospitalized patients in Ege University, Childrens Hospital between the dates.

The frequency of fungi among agents growing in blood culture was evaluated. In case of the recurrent positive cultures from the same patient, only one strain was taken into consideration. Besides, after 15 days of negativity in blood culture, a positive culture was considered as a new candidemia episodes.

Fungi were isolated from the blood cultures using the BacktAlert system (bioMérieux, France). They were identified with conventional mycological methods and their assimilation profiles were determined with ID 32 C (bioMérieux, France) between 2008-2014; and identified by MALDI TOFF MS (bioMérieux, France) between 2014-2017.

A total of 281 fungal species from 252 patients were evaluated. $58.4 \%$ of the patients were male, $41.6 \%$ were female and the mean age was 4.5 (0.01-20.08). Candidemia was common in pediatric surgery unit followed by pediatric intensive care, gastroenterology and oncology services (Table 1).

Two hundred seventy five of these strains (97.8\%) were Candida species, while six were other fungi. The most common species of Candida was C. parapsilosis. Of the isolates, $32.4 \%$ (91/281) were C. albicans and $65.4 \%$ (184/281) were nonalbicans Candida; C. parapsilosis (41.6\%), C. glabrata (6.4\%), C. tropicalis (5.3\%), C. krusei (2.8\%), C. kefyr (2.1\%) and other Candida species (7.2\%) (Table 2).

Table 1. Distribution of fungi by pediatric units

\begin{tabular}{l|}
\multicolumn{1}{|c|}{ Units } \\
\hline Surgery \\
\hline Intensive care \\
\hline Gastroenterology \\
\hline Oncology \\
\hline Haemotology \\
\hline Newborn \\
\hline Bone Marrow Transplantation \\
\hline Immunology \\
\hline Cardiology \\
\hline General pediatri \\
\hline Endocrinology \\
\hline Chest Disease \\
\hline Neurology \\
\hline Infectious disease \\
\hline Nephrology \\
\hline Metabolism \\
\hline Rheumatology \\
\hline Total \\
\hline
\end{tabular}

\begin{tabular}{|c|c|}
\hline Number & $\%$ \\
\hline 64 & 22.8 \\
\hline 48 & 17.1 \\
\hline 46 & 16.4 \\
\hline 29 & 10.9 \\
\hline 25 & 8.9 \\
\hline 17 & 6 \\
\hline 15 & 5.3 \\
\hline 5 & 1.8 \\
\hline 5 & 1.8 \\
\hline 5 & 1.8 \\
\hline 4 & 1.4 \\
\hline 4 & 1.4 \\
\hline 4 & 1.4 \\
\hline 3 & 1.1 \\
\hline 3 & 1.1 \\
\hline 2 & 0.7 \\
\hline 2 & 0.7 \\
\hline 281 & 100 \\
\hline
\end{tabular}

Table 2. Frequency of fungi isolated from blood cultures in pediatric patients during 2008-2017

\begin{tabular}{|l|c|c|}
\hline Isolated Fungi & Number of isolated strains & $\%$ \\
\hline Candida parapsilosis & 117 & 41.6 \\
\hline Candida albicans & 91 & 32.4 \\
\hline Candida glabrata & 18 & 6.4 \\
\hline Candida tropicalis & 15 & 5.3 \\
\hline Candida krusei & 8 & 2.8 \\
\hline Candida kefyr & 6 & 2.1 \\
\hline Candida spp. & 6 & 2.1 \\
\hline Candida guilliermondii & 5 & 1.8 \\
\hline Candida inconspicua & 2 & 0.7 \\
\hline Candida lusitaniae & 2 & 0.7 \\
\hline Candida pelliculosa & 2 & 0.7 \\
\hline Candida dubliniensis & 1 & 0.7 \\
\hline Candida famata & 1 & 0.4 \\
\hline Candida sake & 1 & 0.4 \\
\hline Cryptococcus neoformans & 1 & 0.4 \\
\hline Geotrichum candidum & 1 & 0.4 \\
\hline Geotrichum capitatum & 2 & 0.4 \\
\hline Trichosporon asahii & 1 & 0.4 \\
\hline Zygosaccharomyces spp. & 1 & 0.4 \\
\hline Total & 281 & 100 \\
\hline
\end{tabular}

As a conlusion, although $C$. albicans was the most common species in the first two years of this period, C. parapsilosis took the first place in the following years.

Taking infection control measures into consideration can reduce the incidence of candidemia due to $C$. parapsilosis 